\title{
Topographic visually evoked potentials induced by stereoptic stimulus
}

\author{
SAKUKO FUKAI \\ From the Department of Ophthalmology, Kawasaki Medical School, Kurashiki, Japan 701-01
}

SUMMARY Topographic processes of brain activity during stereopsis were investigated by means of two different principles, with a real stereo target and a computer stereogram. Use of either principle produced the same tendency: an electrically negative focus started from the central region of the scalp and moved to the parietal and occipital regions. These flows of excitation were seen during a period of 90 to $170 \mathrm{~ms}$. The difference between these two stimulus represented a return of the negative focus from the occipital pole to the parietal region in the real stereo target and a spread of the negative focus to the temporal region in the computer stereogram. Since monocular viewing of a real stereo target produces a similar visually evoked potentials wave form but with less intensity, the negative focus in binocular viewing may be due to the enhancement of binocular cells and disparity sensitive neurons in a wide area of the brain cortex. Thus stereoptic brain responses start from the central and parietal regions and move to the occipital region, making a flow of excitation.

Brain activity during a stereoptic vision was studied by means of the newly developed dynamic topography ${ }^{12}$ of visually evoked potentials (VEP). For stereoptic stimulation two different stimulators, a real stereo target and a computer stereogram, were employed and the results were compared.

In the previous studies on stereoptic VEP ${ }^{3-8}$ the main emphasis of discussion was VEP enhanced by a stereoptic target. However, the present paper reports an investigation of the topographic process of brain activity during stereopsis by means of the dynamic topography developed by Lehmann et al.' and Tsutsui et al. ${ }^{2}$

\section{Methods and materials}

Two types of stereo stimulators were used. The first was the combination of a liquid crystal shutter and a real stereo target which has been previously reported. ${ }^{47}$ Electric liquid crystal shutters were used as a visual stimulator with a computer trigger. The stimulus consisted of the appearance of a target for $1000 \mathrm{~ms}$ and its disappearance for $1000 \mathrm{~ms}$. The time needed for the shutter opening was $1 \mathrm{~ms}$ and for its closing $4 \mathrm{~ms}$. VEPs were recorded during the target's appearance for $512 \mathrm{~ms}$. Styrofoam blocks of random

Correspondence to Sakuko Fukai. height, with a surface measurement of $12 \times 12 \mathrm{~mm}$, were fixed on the inside of a transparent plastic perimeter with a diameter of $60 \mathrm{~cm}$. The total number of blocks was 169 , these occupying $20^{\circ}$ of the central field. The average depth of the uneven surface was $4.5 \mathrm{~cm}$ and the distance from the subject's eyes was about $45 \mathrm{~cm}$.

The second stereo stimulator was a red-green stereogram on the cathode ray tube (13 inches $(33 \mathrm{~cm}))$ of a personal computer (NEC PC-8800). The stereogram consisted of three simple rectangles $(4.5 \times 9.0 \mathrm{~cm}, 3.2 \times 6.4 \mathrm{~cm}, 2.3 \times 4.6 \mathrm{~cm}$, with visual angles of $191^{\prime} \times 381^{\prime}, 136^{\prime} \times 271^{\prime}, 98^{\prime} \times 195^{\prime}$, respectively) with disparities of $42^{\prime}, 31^{\prime}$, and $19^{\prime}$ when the shift appeared. Using red-green glasses, the subjects were seated $50 \mathrm{~cm}$ from the CRT.

The computer triggered a shift of rectangles to produce depth in the front of the background. The modes of depth stimuli appeared for $1300 \mathrm{~ms}$ and disappeared for $1300 \mathrm{~ms}$, but VEPs were recorded for only $512 \mathrm{~ms}$ during the appearance time.

The computer-triggered shift of disparity induced a depth perception in front of the background, while the disappearance of disparity showed loss of depth perception. For fixation the subjects were advised to look at the area displaying a disparity, since pinpoint fixation lost the depth sensation. Eye movement was always monitored from electrodes $F p_{1}$ and $F p_{2}$ to 

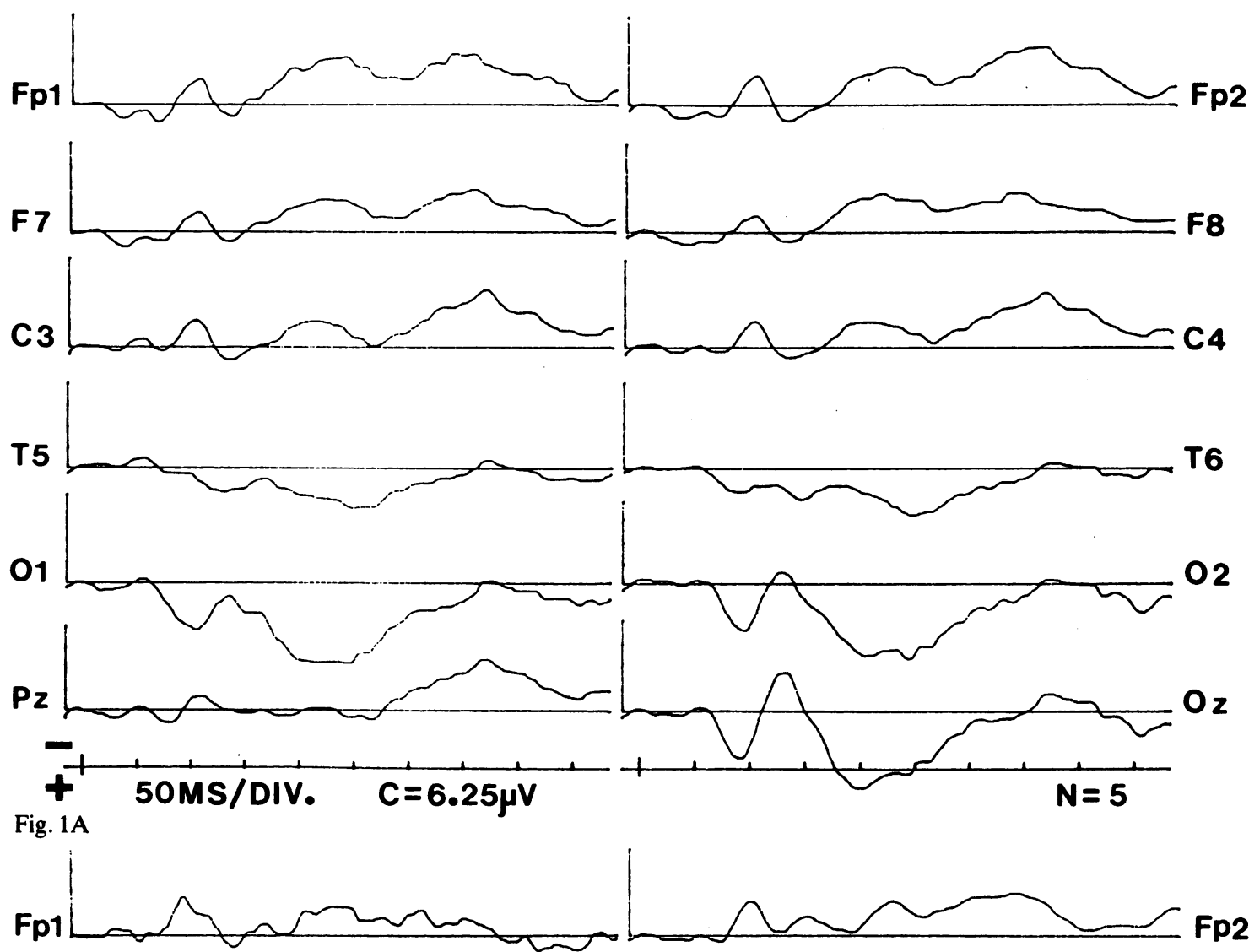

Fp2
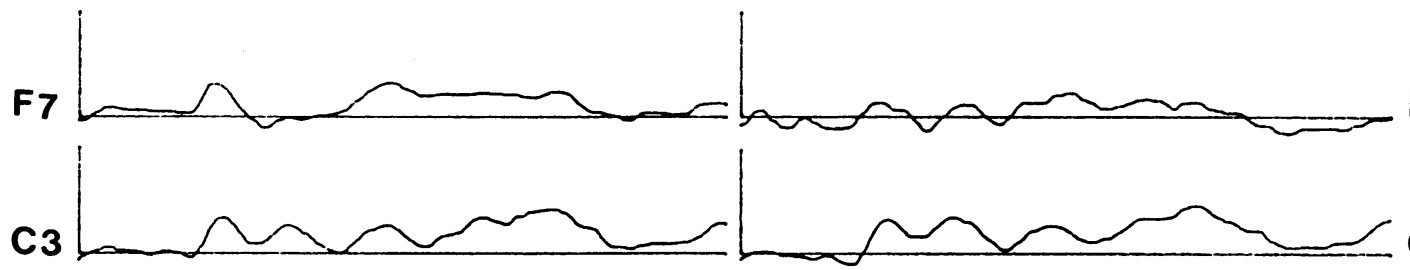

$\mathrm{C} 4$

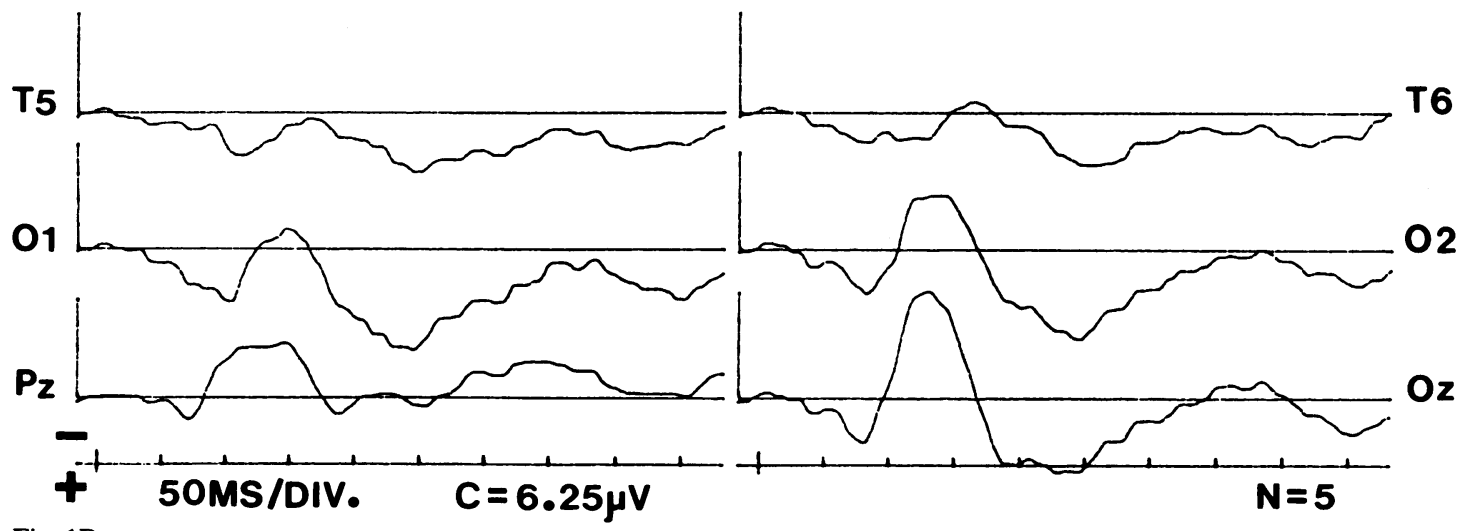

Fig. 1B

Fig. 1 VEPs induced by real stereo target. Grand averaged data from five subjects by monocular (A) and binocular (B) stimulation. 


\section{REAL STEREO Normal Grand average $\quad \mathrm{N}=5$}

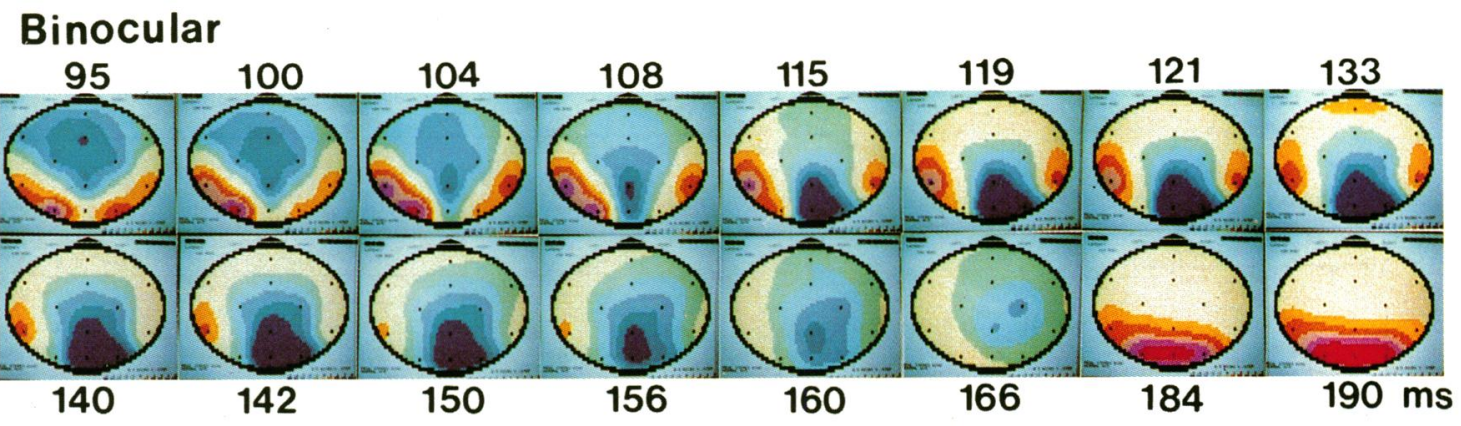

\section{Monocular}

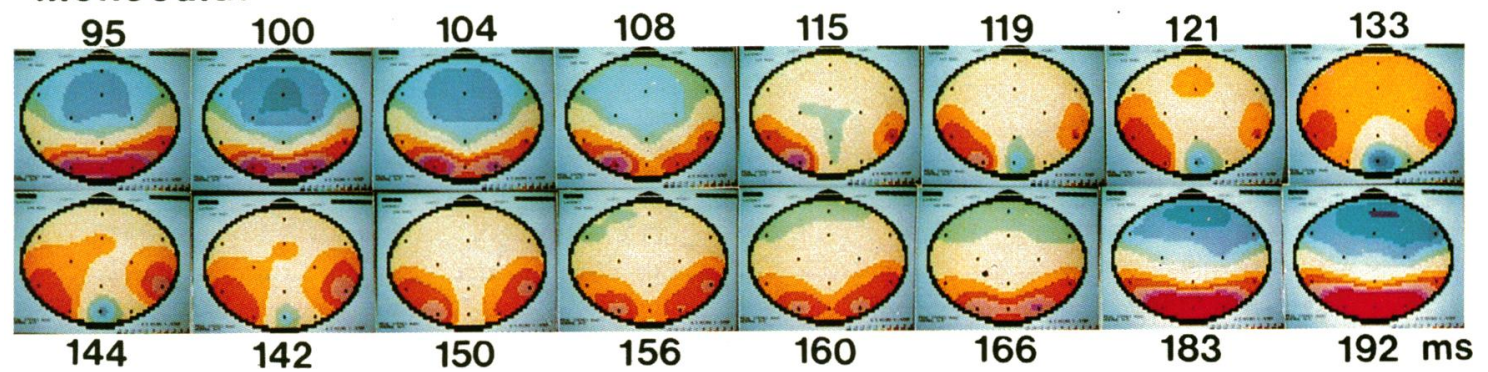

Fig. 2 Grand averaged dynamic topograms of VEPs $(n=5)$ in real stereo target. Red-yellow: positive charge. Green-blue: negative charge. Colour step $0.5 \mu \mathrm{V}$.

avoid contamination of the electro-oculograph (EOG). Blinks were inhibited during the appearance of disparity and permitted during its disappearance.

Recordings were made from 12 electrodes located at $\mathrm{Fp}_{1}, \mathrm{Fp}_{2}, \mathrm{Fz}, \mathrm{F}_{7}, \mathrm{~F}_{8}, \mathrm{C}_{3}, \mathrm{C}_{4}, \mathrm{~T}_{7}, \mathrm{~T}_{8}, \mathrm{Pz}, \mathrm{O}_{1}$, and $\mathrm{O}_{2}$ according to the international $10-20$ method. The reference electrodes were attached at $A_{1}$ and $A_{2}$ and the ground was placed on the forehead. The bandpass filter was set from 0.53 to $60 \mathrm{~Hz}$. Electroencephalographs (EEGs) were recorded by a paper recorder and a 16-channel data recorder (TEAC Co., Tokyo, SR-70) during experiments. Both stimulators produced an average of 50 responses. In order to make an animated movie, the whole process of VEP, completed in about $300 \mathrm{~ms}$, was displayed on the CRT of a BEAM (brain electric activity map) computer (EEG Topography System 500, Sanei Sokki, Tokyo) at $1 \mathrm{~ms}$ intervals. Evoked potentials at each electrode were also recorded by XY recorder.

Ten normal subjects with a stereoacuity of 40 seconds ( 5 males and 5 females, aged 20 to 60 ) were studied and their VEPs were superimposed. For making a dynamic topogram five cases showing a high reproducibility and few electric artefacts were selected for the grand average presented in this paper.

\section{Results}

REAL STEREO TARGET

Major differences in the wave forms of binocular and monocular stimulation were a remarkable enhancement of the $\mathrm{N}_{150}$ component at $\mathrm{Oz}$ and a negative deflection $(-3.4 \mu \mathrm{V})$ from 90 to $175 \mathrm{~ms}$ at $\mathrm{Pz}$ during binocular viewing (Figs. 1A, B). These peculiarities were clearly demonstrated in the dynamic topograms recorded from $95 \mathrm{~ms}$ of binocular viewing. A large negative focus expanded from the $\mathrm{Fz}$ and moved to $\mathrm{Cz}$ (at $100 \mathrm{~ms}$ ), $\mathrm{Pz}$ (at $108 \mathrm{~ms}$ ), and $\mathrm{Oz}$ (at $119 \mathrm{~ms}$ ), continuously, with an amoeba-like movement (Fig. 2, binocular). The negative focus at the occipital pole was reduced in intensity at one time, but its return from the occipital pole to the parietal region was recognised during the period from 136 to 160 ms.

During monocular stimulation, however, the negative focus from 90 to $108 \mathrm{~ms}$ appeared at the $\mathrm{Fz}$ and $\mathrm{Cz}$ (at $108 \mathrm{~ms}$ ), and less intensive development occurred in the parieto-occipital area (Fig. 2, monocular). The later movement of focus which was recognised in the binocular viewing did not appear. The negative focus at the parietal centre $(\mathrm{Pz})$ was peculiar in the binocular viewing, and the increased 


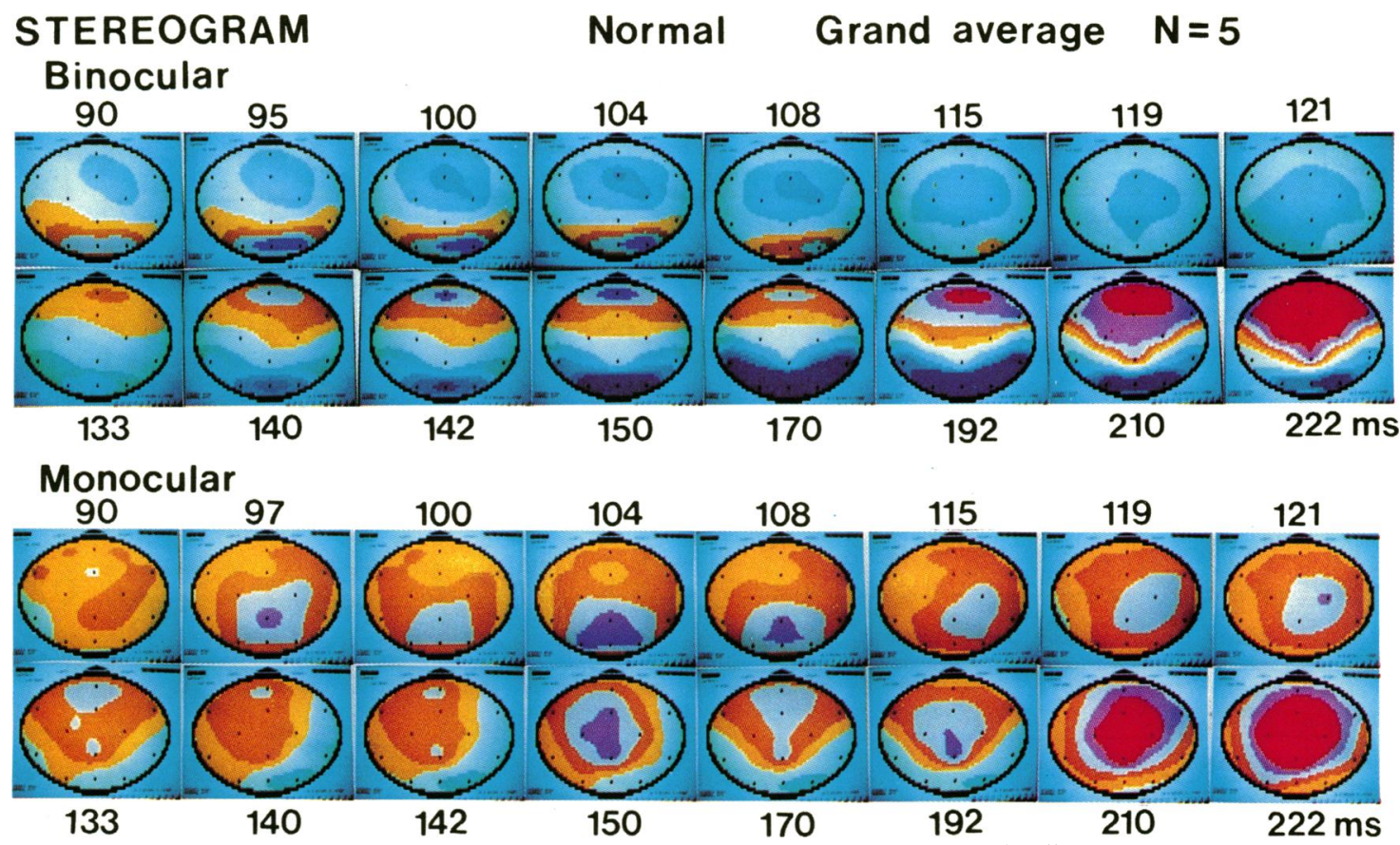

Fig. 3. Grand averaged dynamic topograms of VEPs $(n=5)$ in computer stereogram. Red-yellow: positive charge. Greenblue: negative charge. Colour step $0 \cdot 3 \mu \mathrm{V}$.

negativity at the occipital centre $(\mathrm{Oz})$ developed the enhancement of binocular effect. The amplitude of $\mathrm{N}_{150}$ in monocular viewing was $-2.8 \mu \mathrm{V}$ and in binocular one was $-6 \cdot 6 \mu \mathrm{V}$; thus the binocular effect was $235 \%$. After the parieto-occipital negativity, positive focus appeared in both monocular and binocular viewing for the same period and to the same extent in the occipital region.

\section{COMPUTER STEREOGRAM}

The dynamic topogram (Fig. 3) was displayed from the grand average of five cases whose 12 channel VEPs are illustrated in Figs. 4A, B. From $90 \mathrm{~ms}$ after the appearance of depth a positive discharge appeared in the occipital region and continued up to $108 \mathrm{~ms}$. This response was obtained through both monocular and binocular stimulation and was interpreted as the $\mathrm{P}_{100}$ of conventional VEP. Binocular stimuli, however, showed a peculiar course of responses, that is, a negative focus $(-0.9 \mu \mathrm{V}, 90 \mathrm{~ms})$ appeared from the centroparietal region, moving to the occipital pole with an amoeba-like movement. The maximum occipital focus $(-4.5 \mu \mathrm{V})$ was seen from 170 to $192 \mathrm{~ms}$ and disappeared after $222 \mathrm{~ms}$. This peculiar course was not produced by the monocular stimuli.

\section{Discussion}

For studies on the topographic process of stereopsis in the brain two different stereo stimulators- the real stereo target and the computer stereogram-were used, and results were compared to find out the similarity in stereoptic brain activity.

Both stimulators showed the same tendency: the appearance of a negative focus in the centroparietal region from 90 to $170 \mathrm{~ms}$ after the appearance of depth. The flow of negative focus started from the central portion and moved to the occipital pole with the use of either stimulus. The only difference noted between the effects of the two stimulators was a return of negative focus from the occipital pole to the parietal region with the real stereo target and a spread of negative focus to the temporal region with the computer stereogram.

The flow of negative focus suggested that a relatively wide area of the brain contributes to the establishment of stereopsis. Fusion, convergence, and small eye drift might be necessary to achieve stereopsis. In my present assessment this negative focus is not specific for stereopsis but, rather, it may be the enhancement of the monocular response. This assessment is supported by the observation of a weak 


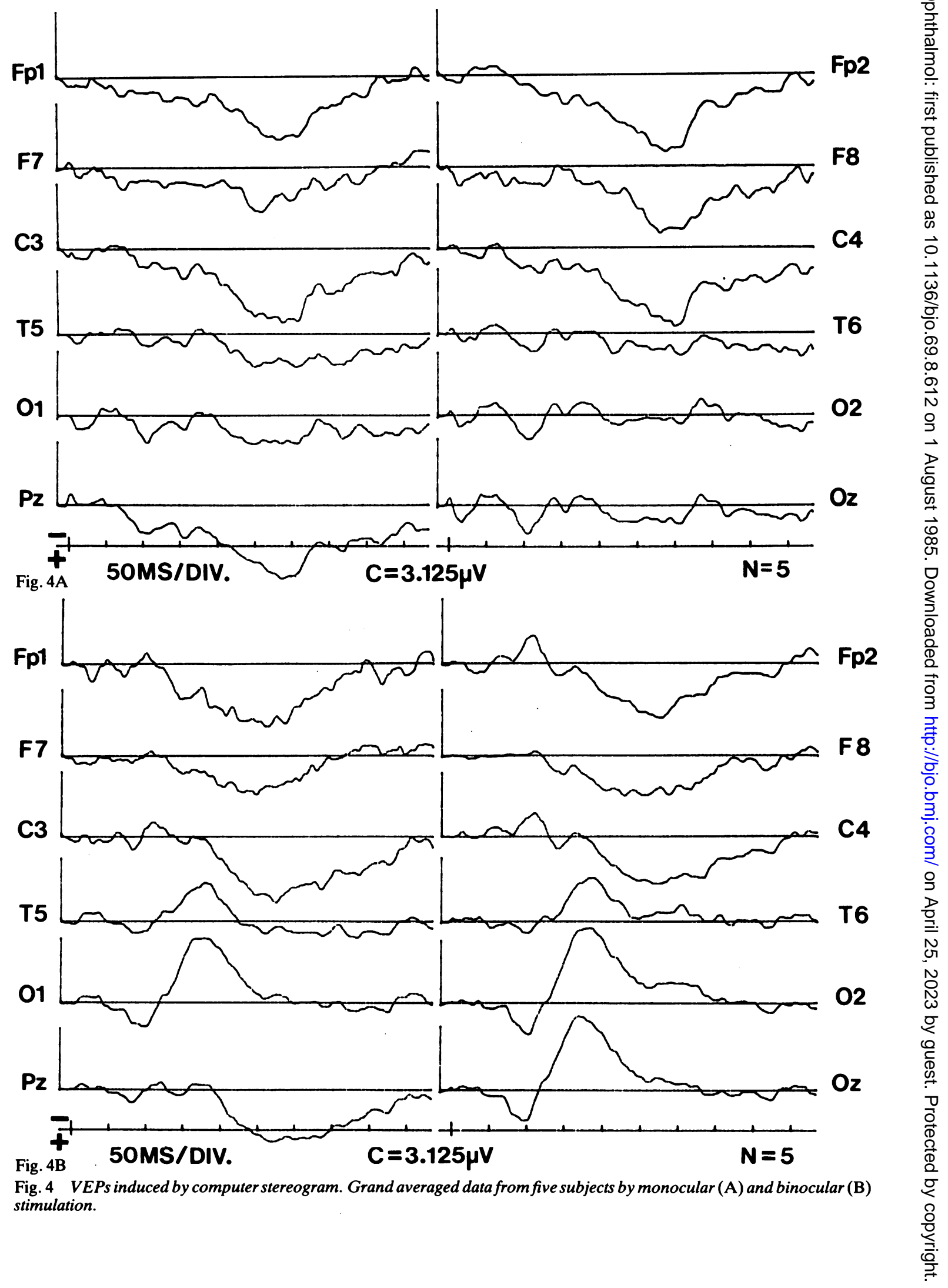


negative focus which appeared in the middle central scalp from 90 to $110 \mathrm{~ms}$ even with the monocular cue of the real stereo target. But the late response in the area from the parietal to the occipital pole was much stronger with binocular stimulation than with monocular. This phenomenon may be due to the enhanced response of binocular cells, which are also sensitive to monocular stimulation to a less extent. Poggio and Fischer' reported that in monkeys nearly all cortical neurons in areas 17 and 18 were binocular neurons, but monocular stimulation with some ocular balance could also occur.

The binocular effect of VEP reported previously depends on the negative component at $150 \mathrm{~ms}\left(\mathrm{~N}_{150}\right)$ in the parietal and occipital areas. In our previous studies on the stereoptic stimulator using the binocular liquid crystal shutters and a real stereo target $^{2478}$ the binocular effect of VEP was parallel to the subjective stereopsis in normal and strabismic patients. In the reports of most other authors ${ }^{356}$ studying the stereoptic VEP a random dot stereogram was employed. As stated earlier, the stereogram used in this investigation did not employ random dots; linear rectangles with disparities were displayed. In the Julesz's type random dot stereogram the appearance of checkerboard-like rectangles might induce a pattern VEP combined with depth discrimination.

As regards brain activity during stereopsis, Herpers et al. ${ }^{6}$ emphasised that the negative component was localised in the central and parietal regions during the period from 150 to $250 \mathrm{~ms}$. Although the peak time of the negative component obtained in this study showed little difference from that of Herpers et al., the same tendency of response was clearly demonstrated here. Herpers et al. emphasised that the stereoptic brain response was localised in the central and parietal region and was not generated in the occipital region. In this study, however, the occipital region showed a negative potential in the late stage. The meaning of the centro- parieto-occipital travel of brain activity has not clearly been interpreted, but it suggests that many steps of neural function may produce stereopsis.

As regards the cerebral centre of stereopsis, no hemisphere preference was found in this study. This result is in agreement with the report of Lehmann and Julesz. ${ }^{3}$ As to the higher integration potentials, such as vertex potentials of $\mathbf{P}_{200}$ and event-related potential $\mathbf{P}_{300}$, the negative component in stereopsis was easily differentiated.

In conclusion, our study shows that the stereoptic response in the brain starts from the central and parietal regions and moves to the occipital region, making a flow of excitation.

This work was presented in part at the Fifth International Orthoptic Congress (1983) in Cannes.

I am extremely grateful to Professor Jun Tsutsui for his valuable instruction, and I thank Ms Matsuda and Ms Hayakawa for technical assistance.

\section{References}

1 Lehmann D, Males HP, Mir Z. Average multichannel EEG potential fields evoked from upper and lower hemi-retina: latency differences. Electroencephalogr Clin Neurophysiol 1977; 43: 725-31.

2 Tsutsui J, Ichihashi K, Kimura H. Dynamic topography of visual evoked potentials and extrageniculate projection in case of Riddoch phenomenon. Jpn J Ophthalmol 1984; 28: 20-30.

3 Lehmann D, Julesz B. Lateralized cortical potentials evoked in humans by dynamic random-dot stereograms. Vision Res 1978; 18: $1265-71$.

4 Fukai S, Abe T, Tsutsui J. Studies on the binocular disparity sensitive VECP. Nippon Ganka Gakkai Zasshi 1980;84: 1629-33.

5 Julesz B, Kropfl W, Petrig B. Large evoked potentials to dynamic random-dot correlograms and stereograms permit quick determination of stereopsis. Proc Natl Acad Sci USA 1980; 77: 2348-51.

6 Herpers MJ, Caberg HB, Mol JMF. Human cerebral potentials evoked by moving dynamic random dot stereograms. Electroencephalogr Clin Neurophysiol 1981; 52: 50-6.

7 Fukai S. Binocular facilitation of VEPs by real stereo-target. Kawaskai Med J 1981; 7: 211-22.

8 Tsutsui J, Kimura H, Fukai S. Binocular facilitation of VECP and binocular disparity. Doc Ophthalmol Proc Ser 1982; 31: 399-406.

9 Poggio GF, Fischer B. Binocular interaction and depth sensitivity in striate and prestriate cortex of behaving rhesus monkey. J Neurophysiol 1977; 40: 1392-404. 